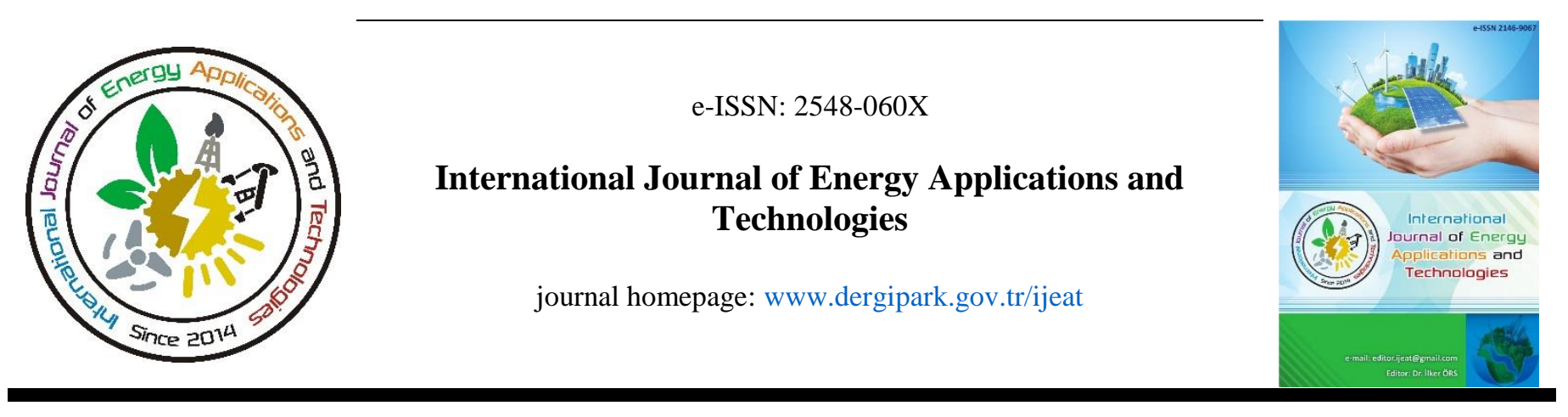

Original Research Article

\title{
A multivariate nonlinear regression model for the resistance power of a light rail vehicle
}

\author{
Mine Sertsöz ${ }^{*}$, Mehmet Fidan \\ Vocational School of Transportation, Eskisehir Technical University, 26140, Eskisehir, Turkey
}

\author{
ARTICLE INFO \\ * Corresponding author \\ msertoz@eskisehir.edu.tr \\ Received September 23, 2020 \\ Accepted February 9, 2021 \\ Published by Editorial Board \\ Members of IJEAT \\ (C) This article is distributed by \\ Turk Journal Park System under \\ the CC 4.0 terms and conditions. \\ doi: 10.31593/ijeat.798799
}

\begin{abstract}
In Turkey, approximately $20 \%$ of the energy expended is spent on transportation. Light rail transportation technology is still in an evolving process. In this development process, it is crucial to have information about the energy consumption of the light rail vehicle according to the different situations of both the vehicle and the railway.

It is necessary to predict the power losses that will occur under different driving conditions sensitively to ensure energy efficiency in light rail systems. The most important of these power losses is the resistance loss caused by contact with the route. Resistance loss is dependent multiple environmental conditions. The most important of these conditions can be listed as the weight of the light rail vehicle, the instantaneous speed of the vehicle, the curve of the route, the ramp slope of the route and the friction force arising from these conditions. Resistance loss is proportional and linearly dependent to some of these variables while others show reverse or nonlinear dependence. Due to these different types of dependencies, it is seen that a single multivariate nonlinear model is needed to explain the loss of resistance in all different conditions. In this study, a new and accurate model for resistance losses has been developed by fitting numerical values obtained from different scenarios to multivariate nonlinear regression model.
\end{abstract}

Keywords: Energy efficiency; Nonlinear regression; Optimization; Railway

\section{Introduction}

It is not enough to just focus on production for today's rapidly developing technology. Efficient use of technology is more important than production for both financial and environmental resources. Technology needs efficient use of resources to be sustainable. The methods applied for efficiency management in rail systems in Turkey is seen as inadequate in terms of sustainability. Depending on the developments in the field of rail systems, if the efficiency studies in this field increase, both the financial accumulation of the country can be increased and the positive effects of reducing energy use on the environment can be observed.

There are several studies in the literature on the efficiency of rail systems. Since this study deals with a light rail vehicle, the case studies on the efficiency of light rail vehicles in the literature are mentioned, as well as some examples of single studies on different energy management systems.

In the project named MALTESE, energy consumption calculations of the existing LRT systems for energy management and efficiency in Light Rail Systems in Europe were made, in addition to this, a new vehicle with the energy balance archetype (EPA) Model was developed [1]. In another study, train energy consumption model was developed according to instantaneous regenerative braking efficiency and the developed model was calibrated with an unrestricted nonlinear optimization procedure using data from Portland, Oregon [2]. In the related study, it has been explained that energy recovery reduces the total power consumption by $20 \%$ and significantly increases the energy efficiency of the system. This study applies to project-level analysis and can come to the energy efficiency planning 
stage. The results also show that the proposed modelling approach can capture the energy consumption differences associated with trains, routes, and operational characteristics. In rail systems, the instantaneous deceleration level is related to energy regeneration and this can be used in railway crossing energy modelling. Mittal suggested an analytical method to estimate energy consumption related to speed, passenger load and train configuration accurately [3]. Mittal's study has two critical disadvantages. Firstly, Mittal's model only depends on average speed (exist of fluctuations) for energy prediction. Secondly, Mittal's model is lack of energy regeneration.

In the literature, models used for energy optimization have disadvantages due to insufficiency in instant energy prediction [4-9]. A passenger rail simulation framework, which incorporated route and train characteristics, speed, passenger load and regenerative braking into the energy modelling practice, was designed by The National Cooperative Rail Research Program (NCRRP) [10]. The design of the NCRRP is an excel-based tool and could not be implemented in complex frameworks, such as smartphone eco-driving, eco-routing systems and traffic simulation software. In addition, the energy prediction within the framework uses only average speed and a constant regenerative efficiency. Other studies have complexity in model specification so they are not suitable for intelligent transportation system (ITS) applications [11-13]. These models cannot be used especially for road electric vehicles. There have been numerous studies on modelling rail electric consumption but as mentioned they are not sufficient for having some disadvantages [14-18].

In one of the related studies, a method has been proposed to identify the parameters of the simplified running resistance formula for different types of trains by utilizing telemetric data that are available for almost all railway companies [19]. The method of Aradi, Becsi and Gaspar can determine the parameters of the formula from historical data in an offline way. Since the method considers the inclinations of the railway tracks any runs recorded can be used for the evaluation of the parameters in contrast with the classic methods that need tracks with constant grades or special test cases.

Spinalbese expressed running resistance as a multiplication of mass and second degree polynomial function of speed of a train to use in CFD analysis for aerodynamic resistances [20]. Michalek expressed train resistance with multiplication of the weight of the train and a second degree polynomial of speed for a container train [21]. Kwon modelled resistance of high speed train with second degree polynomial of speed, where the coefficients of the polynomial depends on mass of train, dimensionless parameter related to type of rolling stock, front surface cross section area, parameter for shape of the train, parameter for condition of the surface and partial perimeter of the rolling stock down to rail level [22]. Both resistance models of Spinalpese, Michalek and Kwon do not include the effects of curve and ramp of the road, which are handled by our research.

The structure of this study can be summarized as follow: In the Introduction section, energy efficiency management and its importance in rail systems; applications of energy efficiency management in this area in the world are given. In the Resistance of Light Rail Vehicles Section, resistance for the movement of the light rail vehicles (LRV) and LRV is explained in detail. Theoretical explanation of the model used is given in Multivariate Nonlinear Regression Model Section. How the model is applied to train resistance problem and the problem's equation is given in Proposed Model for Resistance Section. The accuracy tests of the model (such as RMSE and statistical compatibility) are specified in Numerical Results of Proposed Model Section. And lastly, the results, outcomings, and benefits of this study is handled in Conclusion Section.

\section{Resistance of Light Rail Vehicle}

In this study, the ESTRAM LRV is modelled, which is shown in Fig.1. Tram sets of LRV are produced by Bombardier. This LRV has five cars. Five cars are positioned with four joining modules at each end. Each joining module has a bellow in gangway. Vehicle are fitted with three bogie modules. First two cars have motor bogies and third one has a trailer bogie which is not electrically powered. All the bogies have same basic construction. Cars 1, 2, 4 and 5 are equipped with sliding plug doors. All doors are located on the right side of the vehicle according to direction of travel.

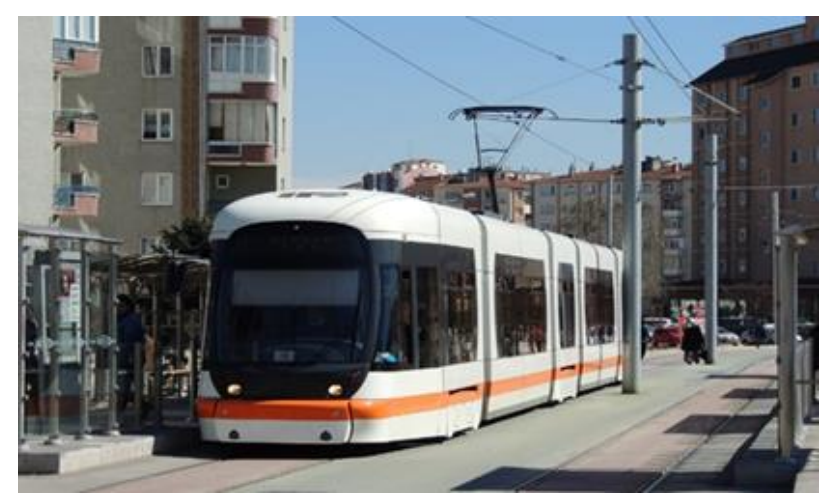

Fig. 1. Eskişehir Light Rail Vehicle [23]

\subsection{Power consumption due to resistance force}

The $\mathrm{P}$ value is the power requirement for continuous regime. $\mathrm{G}_{\mathrm{T}}$ is load of the LRV and there are four types load. These are empty load (AW0), AW0 plus full seated load, including crew (AW1), AW1 plus 4 passengers per $\mathrm{m}^{2}$ (AW2) and AW1 plus 6 passengers per $\mathrm{m}^{2}$ (AW3) for this locomotive. $\mathrm{V}$ is speed, $\mathrm{r}$ is ramp level as \%, $\mathrm{k}$ is radius of curve in terms of 
meter, $\mathrm{n}$ is axle number, $r_{t}$ is cruising resistance, $r_{k}$ is curve resistance and $r_{r}$ is the gradient resistance of LRV set. Summation of all resistances is $\mathrm{T}$ as named Wheel Force. Other resistances as aerodynamic and acceleration resistances are neglected in this study.

$$
\begin{aligned}
& P=\frac{T \times V}{367}(\mathrm{~kW}) \\
& T=r_{t}+r_{k}+r_{r} \\
& r_{t}=\left(0,65+\frac{13 \times n}{G_{T}}+0,01 \times V+\frac{0,031 \times(V+15)^{2}}{G_{T}}\right) G_{T} \\
& r_{k}=\left(\frac{400}{k-20}\right) G_{T} \\
& r_{r}=r \times G_{T}
\end{aligned}
$$

The sum of all resistance types has a linear relationship with power consumption according to Equation (1). The T, which denotes the wheel force, can be increased by either decreasing $\mathrm{V}$ or increasing $\mathrm{P}$, traction power in theoretically. However, the adhesion caused by the wheel force limits the power. Adhesion is dependent to the physical characteristics of the materials used in the structure between rail and wheel, but this study is not concentrated to adhesion factor.

\section{Results and Discussion}

If we assume that $f$ is a nonlinear function chosen for the expectation of $\mathrm{Y}$, then we can generalize the nonlinear regression as Equation (6)[24].

$$
Y=f(\vec{X}, \vec{\beta})+\varepsilon
$$

In Equation (6), $\vec{X}$ denotes the vector of input variables, $\vec{\beta}$ denotes the vector of coefficients of the model to be expected by fitting $\mathrm{Y}$ and $\varepsilon$ is error of the model. The expected value of $\varepsilon$ is assumed as 0 as in linear regression model.

The model parameters defined by $\vec{\beta}$ vector is found by applying least squares method as expressed in the following steps.

1. Define the $\mathbf{P}$ dimensional expectation surface $\eta(\beta)$ in $n$ dimensional response surface for $Y$.

2. Find the closest point $\hat{\eta}$ to $\mathrm{Y}$ by applying Equation (7).

$$
\hat{\eta}=Q^{T} Y
$$

3. Approximate the parameter vector $\vec{\beta}$ by Equation (8).

$$
\widehat{\beta}=R^{-1} Q^{T} \hat{\eta}
$$

For Equations (7) and (8), $\mathrm{X}=\mathrm{QR}$ where $\mathrm{X}$ is a matrix that contains possible $\vec{X}$ vectors in its columns.

\section{Proposed Model for Resistance}

In the proposed model, there are four input variables. These input variables of the multivariate regression model are taken as discrete variables whose possible values are defined as below.
$X_{1}=\mathrm{G}_{\mathrm{T}}:[32.28 ; 43.08 ; 46.455 ; 53.677]$ tone

Number of $\mathrm{G}_{\mathrm{T}}$ types $\left(\mathrm{N}_{\mathrm{GT}}\right): 4$

$X_{2}=\mathrm{V}:[0 ; 10 ; 20 ; 30 ; 40 ; 50 ; 60 ; 70] \mathrm{km} / \mathrm{h}$

Number of $\mathrm{V}$ types $\left(\mathrm{N}_{\mathrm{V}}\right): 8$

$X_{3}=\mathrm{k}:[21 ; 28 ; 35 ; 42] \mathrm{m}$

Number of k types $\left(\mathrm{N}_{\mathrm{K}}\right): 4$

$X_{4}=$ r: $[-7 ; 0 ; 7] \%$

Number of $r$ types $\left(\mathrm{N}_{\mathrm{R}}\right): 3$

$N_{\text {scenario }}=N_{G T} \times N_{V} \times N_{K} \times N_{R}=384$

As shown in Equation (17), 384 four-dimensional input vectors are constructed for 384 possible scenarios.

Four sequential nonlinear regression models are constructed by least square method with bi-square robust weight function [25]. First model is a univariate model, which just depends on $\mathrm{G}_{\mathrm{T}}$ as shown in Equation (18).

$\mathrm{T}_{\text {model1 }}=\beta_{1,1}+\beta_{1,2} \mathrm{X}_{1}^{\beta_{1,3}}$

Second model is a bivariate model, which is multiplication of first model and a model which depends on $\mathrm{V}$ as shown in Equation (19).

$\mathrm{T}_{\text {model } 2}=\prod_{\mathrm{i}=1}^{2}\left(\beta_{\mathrm{i}, 1}+\beta_{\mathrm{i}, 2} \mathrm{X}_{\mathrm{i}}^{\beta_{\mathrm{i}, 3}}\right)$

Third model is a three-variable model, which is multiplication of second model and a model which depends on $\mathrm{k}$ as shown in Equation (20).

$\mathrm{T}_{\text {model } 3}=\prod_{\mathrm{i}=1}^{3}\left(\beta_{\mathrm{i}, 1}+\beta_{\mathrm{i}, 2} \mathrm{X}_{\mathrm{i}}^{\beta_{\mathrm{i}, 3}}\right)$

The fourth and ultimate model is summation of third model and a model which depends on $r$ as shown in Equation (21).

$\mathrm{T}_{\text {model } 4}=\prod_{\mathrm{i}=1}^{3}\left(\beta_{\mathrm{i}, 1}+\beta_{\mathrm{i}, 2} \mathrm{X}_{\mathrm{i}}^{\beta_{\mathrm{i}, 3}}\right)+\left(\beta_{4,1}+\beta_{4,2} \mathrm{X}_{4}^{\beta_{4,3}}\right)$

The optimum parameters found for these for model are listed in Table 1.

Table 1. $\beta_{i, j}$ Coefficients of the resistance model

\begin{tabular}{cccc}
\hline $\boldsymbol{\beta}_{\boldsymbol{i}, \boldsymbol{j}}$ & $\mathbf{j}=\mathbf{1}$ & $\mathbf{j}=\mathbf{2}$ & $\mathbf{j}=\mathbf{3}$ \\
\hline $\mathrm{i}=1$ & 744.7489 & 69.5402 & 1.1244 \\
$\mathrm{i}=2$ & 0.9819 & 0.0001 & 1.4737 \\
$\mathrm{i}=3$ & 0.0547 & $3.4 \cdot 10^{8}$ & -6.0851 \\
$\mathrm{i}=4$ & 2.8687 & 43.8730 & 1 \\
\hline
\end{tabular}

\section{Numerical Results for Proposed Model}

By using just $\mathrm{G}_{\mathrm{T}}$ components of the input vectors, the predictions shown in Fig. 2 can be obtained by first model. This model gives an approximate average resistance under specific load (AW0, AW1,AW2 or AW3). The Model1 shown in Fig.2 does not give an information about the 
resistance oscillations caused by curve and ramp. Moreover, Model1 is lack of inclination information in the resistance behavior caused by increase in speed.

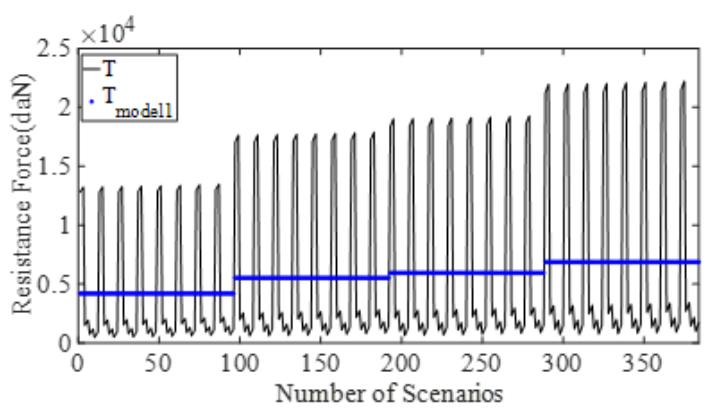

Fig.2. Prediction of Model1 for resistance force

The second model uses $\mathrm{G}_{\mathrm{T}}$ and $\mathrm{V}$ components of the input vectors and find the predictions shown in Fig. 3. The model handles variations not only in load, but also in speed. The addition of the speed information to the model enabled to the catch the trend in the inclined increase of the mean value of the resistance vibration.

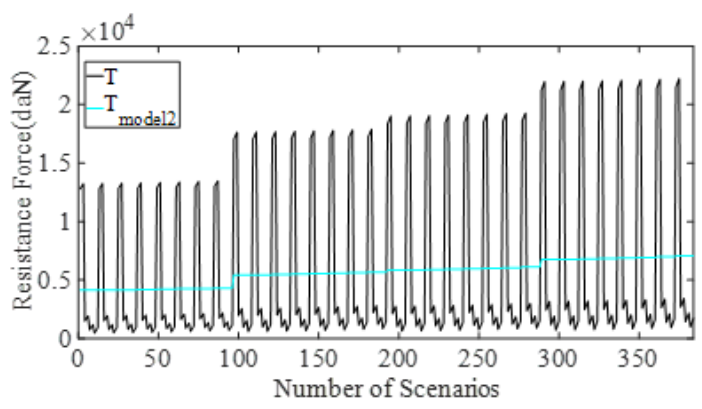

Fig.3. Prediction of Model2 for resistance force

The third model includes effect of curve in the model and obtained the predictions shown in Fig. 4. The third model gives strong information about resistance force which shows that curve is crucial for resistance force. In Fig.4, the oscillations of the resistance can be approximately predicted by including information of the curve.

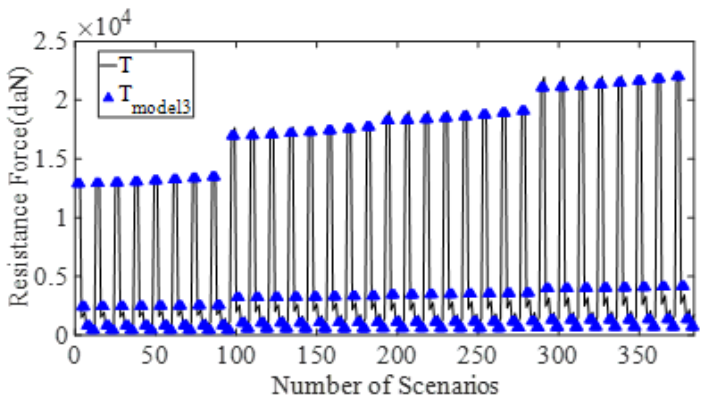

Fig.4. Prediction of Model3 for resistance force

The forth and complete model handles both load, speed, curve and ramp variables and find the predictions shown in Figure 5. This model fine tunes the predictions of third model by using information of the ramp. The Model 4 shown in Fig. 5 has a component of ramp.

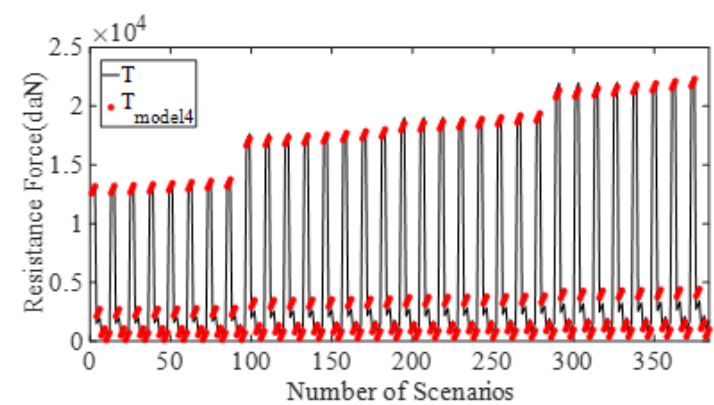

Fig.5. Prediction of Model4 for resistance force

The predictions of Model1 are also scattered in terms of exact values of the resistance force as in Figure 6. Due to the inability of Model1 to catch the resistance oscillation caused by the curve and ramp, the predicted values fell to points far from the exact values and this primitive model shows that load is not sufficient to obtain resistance force as shown in Figure 6.

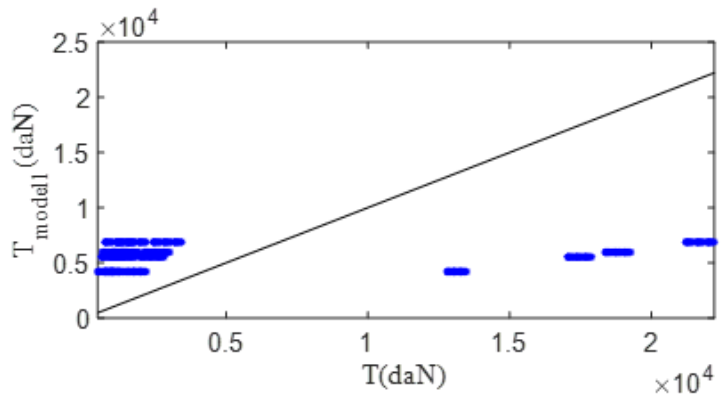

Fig.6. Exact T values vs. predictions of Model1

The predictions of Model2 are scattered in terms of exact values of the resistance force as in Figure 7. As shown in Figure 7, load and speed are not sufficient to obtain resistance force, but this model constructs the backbone of the ultimate model.

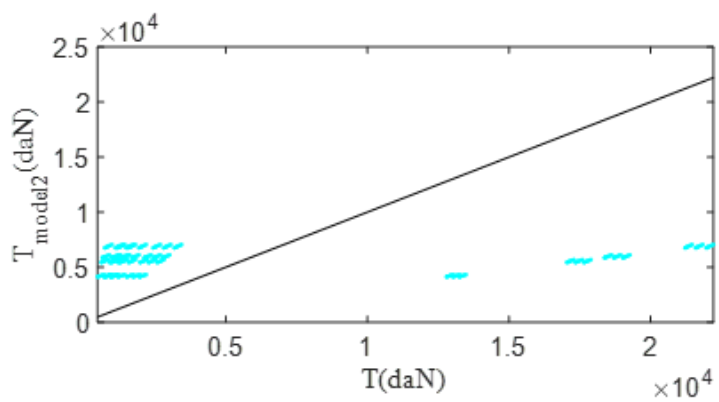

Fig.7. Exact T values vs. predictions of Model2

The predictions of Model3 are scattered in terms of exact values of the resistance force as in Figure 8. Inclusion of the curve information strengthens the model significantly.

The predictions of ultimate model are scattered in terms of exact values of the resistance force as in Figure 8. As shown in Figure 9, ramp fine tunes the predictions of Model3.

The RRMSE\% and MAPE\% of the models are given in Table 2 . 


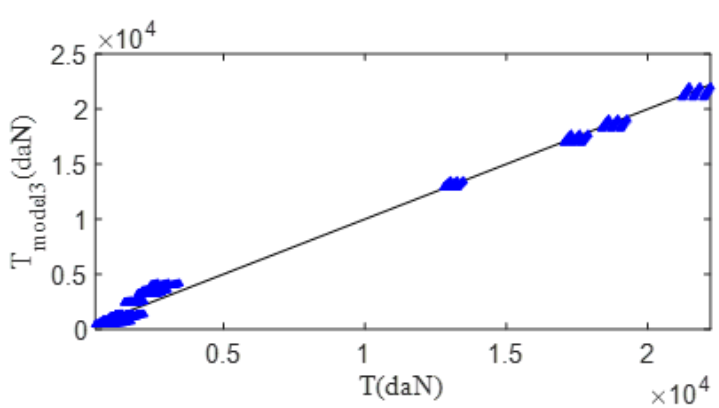

Fig.8. Exact $\mathrm{T}$ values vs. predictions of Model3

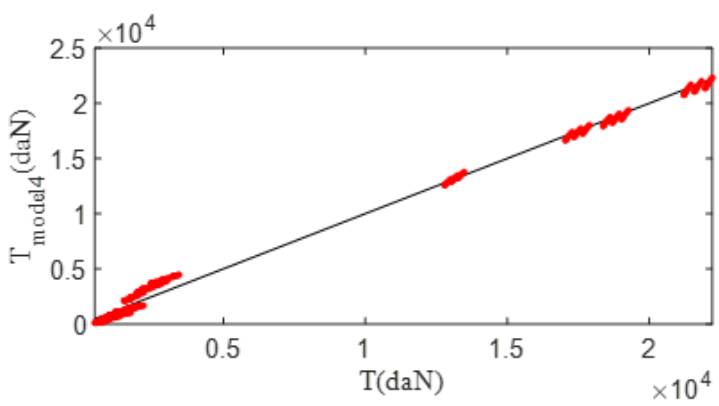

Fig.9. Exact T Values vs. Predictions of Model4

Table 2. RRMSE and MPE of models as percentage

\begin{tabular}{ccc}
\hline Models & RRMSE\% & MPE\% \\
\hline Model 1 & 126.34 & 221.76 \\
Model 2 & 126.33 & 221.60 \\
Model 3 & 10.79 & 5.38 \\
Model 4 & 9.84 & 8.14 \\
\hline
\end{tabular}

When the values in Table 2 are examined, it is seen that the prediction error of Model3 is reduced to reasonable levels and Model4 provides an improvement in the error in terms of RMSE. The correlation of the predictions of the models with exact resistance force are given in Table 3.

Table 3. R of Models

\begin{tabular}{|c|c|}
\hline Models & R \\
\hline Model 1 & 0.1337 \\
\hline Model 2 & 0.1341 \\
\hline Model 3 & 0.9964 \\
\hline Model 4 & 0.9970 \\
\hline
\end{tabular}

When the correlation values in Table 3 are examined, the outputs of Model-1 and Model-2 cannot provide sufficient correlation with the actual values, while Model-3 captures a very strong correlation with the real data due to the oscillation in resistance, and this correlation of Model-4 is further It has been seen to strengthen.

\section{Conclusion}

By conclusion, the proposed model reveals the nonlinear relation of the resistance force with load, speed, curve and ramp. The results obtained by Model 3 and Model 4 shows the crucial role of curve on resistance force. The usage of curve does not only decrease the RMSE, but also increase the correlation of the model with exact $\mathrm{T}$ values significantly.
There is an exponentially inverse relationship with resistance and curve. The multivariate model is a multiplicative model of the models of load, speed and curve, which is improved by summation of ramp model. As a future work, the coefficients of the model can be improved by using NARX-net or ANN and different nonlinear regression models can be tested for obtaining a more sensitive model.

\section{Acknowledgement}

This study has presented as oral at International Conference on Advanced Technologies (ICAT'20), and, it has been published at conference abstract book as abstract.

\section{$\underline{\text { ORCID }}$}

M. Sertsöz

0000-0003-1641-9191

M. Fidan

0000-0003-2883-9863

\section{References}

[1] Krug, S. 2008. Public transport: Energy efficiency of light train systems. SAVE.

[2] Wang, J. and Rakha, H.A. 2017. Electric train energy consumption modeling. Applied Energy, 193, 346-355.

[3] Mittal, R.K. 1977. "Energy intensity of intercity passenger rail. Final report,"; Department of Transportation, Washington, DC (USA). Office of Univ. Research, DOT/RSPD/DPB-50-78/7 United States 10.2172/6300399 Dept. of Transportation, Washington, DC.

https://www.osti.gov/servlets/purl/6300399. (30 March 2021.)

[4] Kim, K. and Chien, S. I.-J. 2011. Optimal train operation for minimum energy consumption considering track alignment, speed limit, and schedule adherence. Journal of Transportation Engineering, 137(9), 665-674.

[5] Sicre, C., Cucala, A.P., Fernández, A. and Lukaszewicz, P. 2012. Modeling and optimizing energy-efficient manual driving on high-speed lines. IEEJ Transactions on Electrical and Electronic Engineering, 7(6), 633-640.

[6] Su, S., Li, X., Tang, T. and Gao, Z. 2013. A Subway Train Timetable Optimization Approach Based on Energy-Efficient Operation Strategy. IEEE Transactions on Intelligent Transportation Systems, 14(2), 883-893.

[7] González-Gil, A., Palacin, R., Batty, P. and Powell, J. 2014. A systems approach to reduce urban rail energy consumption. Energy Conversion and Management, 80, 509-524. 
[8] Li, X. and Lo, H.K. 2014. An energy-efficient scheduling and speed control approach for metro rail operations. Transportation Research Part B: Methodological, 64, pp. 73-89.

[9] Golovitcher, I.M. 2001. Energy efficient control of rail vehicles. IEEE International Conference on Systems, Man and Cybernetics. e-Systems and e-Man for Cybernetics in Cyberspace (Cat. No. 01CH37236), 1:IEEE, 658-663.

[10] Jenks, C.W., Goldstein, L.D., Avery, A.P., Delaney, E.P. and Lamberton, S. 2015. National cooperative rail research program (ncrrp) report 3: Comparison of passenger rail energy consumption with competing modes. Washington (DC): Transportation Research Board.

[11] A. Moawad, N. Kim, N. Shidore, and A. Rousseau, "Assessment of vehicle sizing, energy consumption and cost through large scale simulation of advanced vehicle technologies," Argonne National Lab.(ANL), Argonne, IL (United States), 2016.

[12] Lewis, A.M., Kelly, J.C. and Keoleian, G.A. 2012. Evaluating the life cycle greenhouse gas emissions from a lightweight plug-in hybrid electric vehicle in a regional context. IEEE International Symposium on Sustainable Systems and Technology (ISSST), 2012: IEEE, 1-6.

[13] Lewis, A.M., Kelly, J.C. and Keoleian, G.A. 2014. Vehicle lightweighting vs. electrification: life cycle energy and GHG emissions results for diverse powertrain vehicles. Applied Energy, 126, 13-20.

[14] De Gennaro, M., Paffumi, E., Scholz, H. and Martini, G. 2014. GIS-driven analysis of e-mobility in urban areas: An evaluation of the impact on the electric energy grid. Applied Energy, 124, 94-116.

[15] Rambaldi, L., Bocci, E. and Orecchini, F. 2011. Preliminary experimental evaluation of a four wheel motors, batteries plus ultracapacitors and series hybrid powertrain. Applied Energy, 88(2), 442-448.

[16] Abousleiman, R. and Rawashdeh, O. 2015. Energy consumption model of an electric vehicle. IEEE Transportation Electrification Conference and Expo (ITEC), 2015: IEEE, 1-5.

[17] Rajashekara, K. and Rathore, A.K. 2015. Power conversion and control for fuel cell systems in transportation and stationary power generation. Electric Power Components and Systems, 43(12), 1376-1387.

[18] Wang, J. and Rakha, H.A. 2016. Modeling fuel consumption of hybrid electric buses: Model development and comparison with conventional buses. Transportation Research Record, 2539(1), 94-102.

[19] Aradi, S., Bécsi, T. and Gáspár, P. 2015. Estimation of running resistance of electric trains based on on-board telematics system. International Journal of Heavy Vehicle Systems, 22(3), 277-291.

[20] Spinalbese, A. 2018. An Experimental Method For The Estimation Of The Adhesion Coefficient And The Running Resistance Of A Train. Master of Science in Mechanical Engineering, School Of Industrial And Information Engineering, Politecnico Di Milano.

[21] Michálek, T. 2017. Modification of train resistance formulae for container trains based on operational rundown tests. Proceedings of the Institution of Mechanical Engineers, Part F: Journal of Rail and Rapid Transit, 232(6), 1588-1597, 2017.

[22] Kwon, H. 2017. A study on the resistance force and the aerodynamic drag of Korean high-speed trains. Vehicle System Dynamics, 56(8), 1250-1268.

[23] Hobson, S. 2015. Bombardier Flexity Outlook tram (EsTram - Eskişehir

Tramvay).https://www.flickr.com/photos/30389998@ N03/16742194366/ .(30 March 2021.)

[24] Bates, D.B. and Watts, D.G. Nonlinear regression analysis and its applications, Wiley, New York, 1988.

[25] Dumouchel, W. and O'Brien, F. 1989. Integrating a robust option into a multiple regression computing environment. Computer science and statistics: Proceedings of the 21st symposium on the interface, American Statistical Association Alexandria, VA, 297302.

NOTE: This article was presented at International Conference on Advanced Technologies (ICAT'20). It was evaluated by 2 Reviewers before publish, and it has been passed all publication and evaluation processes of journal. 